\title{
Biochemical and Molecular Diversity in the Chilean Strawberry and its Implications for Plant Breeding \\ V. Becerra ${ }^{1}$ and M. Paredes
}

INIA, Centro Regional de Investigación Quilamapu, Casilla 426, Chillán, Chile

\author{
A. Lavín \\ INIA, Centro Experimental Cauquenes, Casilla 165, Cauquenes
}

The Chilean wild strawberry (Fragaria chiloensis L. Duch.) is distributed in a wide range of agroecological systems, and as a result, has evolved considerable diversity in morphological and molecular traits. The native and ancient land races of $F$. chiloensis could be used as parents in a breeding program to improve the species or as a gene pool to improve the cultivated strawberry $F$. ×ananassa. At the national Research Institute of Agriculture (INIA), Experimental Center in Cauquenes, a collection of wild strawberry germplasm sampled throughout the country (Cameron et al., 1993) is maintained. Over the last ten years, the morphological and molecular patterns of variability in these collections have been examined (Becerra et al., 2001, 2002; Maureira et al., 1996). Here we describe the patterns in genetic diversity of selected Chilean strawberry accessions using isozymes and amplified fragment lenght polymorphism (AFLP).

\section{Materials and Methods}

Leaf sample preparation for isozyme analysis were similar to those described by Arulsekar et al., 1981. DNA was extracted from 61 genotypes representing the geographic and climatic range of strawberries in Chile (Table 1). DNA was extracted as previously described (Becerra and Gepts, 1994) and was amplified using the procedure of Becerra et al., 2001. The following primer pairs were utilized: EcoRIAGC/MseI-CTG; EcoRI-ACA/MseI-CAG; EcoRI-ACC/MseI-CAA; EcoRI-ACC/MseICAT; EcoRI-ACT/MseI-CAC y EcoRI-AGG/ $M s e I-C T T$. The gels were run according to Vos et al., 1995, with a nonradioactive modification. Band fragments were scored as present (1) or absent (0) and analysed using UPGMA clustering of Jaccard's coefficient (NTSYS) version 2.1, Rohlf, 2000.

\section{Results and Discussion}

The isozyme work showed a very low level of diversity in three isozyme systems, glucose phosphate isomerase (GPI), leucine aminopeptidase (LAP) and phosphoglucomutase (PGM). Only a few accessions showed any polymorphism. However, a great deal of diversity was previously found among genotypes for morphological traits. For example, growth habit type varied from postrate to erect, leaf width range between 2.42 and $4.32 \mathrm{~cm}$, and runner first node length varied between 19.0

Research financed through FONDECYT Project 1980166.

1E-mail vbecerra@quilamapu.inia.cl.
Table 1. Chilean accessions analyzed with biochemical and molecular markers.

\begin{tabular}{|c|c|c|c|c|c|}
\hline Location & Province & Accession & $\begin{array}{c}\text { South } \\
\text { latitude }\end{array}$ & $\begin{array}{c}\text { West } \\
\text { longitude }\end{array}$ & $\begin{array}{l}\text { Altitude } \\
\text { (MASL) }\end{array}$ \\
\hline Ambato & Ecuador & 96 AMB1A & 01.01 & $\mathrm{NI}^{\mathrm{z}}$ & 2500 \\
\hline Iloca & Curicó & 92ILO1A & 34.55 & 72.11 & NI \\
\hline Vilches & Talca & 3VIL1A & 35.34 .13 & 70.37 .34 & 535 \\
\hline Huelón & Talca & 3HUE1A & 35.05 .75 & 72.03 .55 & 50 \\
\hline Carrizal & Talca & 3RRI1A & 35.16 .45 & 72.14 .20 & 245 \\
\hline Curanipe & Cauquenes & 3CUR1A & 35.51 .55 & 72.37 .00 & 165 \\
\hline Cobquecura & Ñuble & $3 \mathrm{COB} 1 \mathrm{~A}$ & 36.12 .20 & 72.47 .42 & 155 \\
\hline Río los Sauces & Ñuble & 90SAU1A & 36.25 & 71.08 & NI \\
\hline Termas de Chillán & Ñuble & $3 \mathrm{TCH} 5 \mathrm{~A}$ & 36.54 .27 & 71.24 .54 & 1500 \\
\hline Los Lleuques & Ñuble & 3LLE1A & 36.52 .06 & 71.35 .87 & 1000 \\
\hline Laguna Laja & Biobío & $3 \mathrm{LAJ} 2 \mathrm{~A}$ & 37.23 .74 & 71.24 .52 & 1020 \\
\hline Purén & Angol & 97PUR1A & 37.57 & 73.10 & NI \\
\hline Cayucupil & Arauco & 3CAY5A & 37.48 .18 & 73.09 .34 & 660 \\
\hline Ramadilla & Arauco & 3RAM1A & 37.20 .21 & 73.13 .58 & 240 \\
\hline Quilapo & Arauco & 3APO1A & 37.24 .47 & 73.33 .74 & 180 \\
\hline Trongol & Arauco & 3TRO1A & 37.33 .59 & 73.21 .17 & 130 \\
\hline Cañete & Arauco & 3CAÑ1A & 37.37 .49 & 73.25 .02 & 160 \\
\hline Cayucupil & Arauco & 94CAY1A & 37.44 .40 & 73.35 .50 & NI \\
\hline Elicura & Arauco & 3ELI1A & 37.48 .19 & 73.09 .34 & 325 \\
\hline Agua fría & Malleco & 3FRI1A & 37.46 .02 & 72.48 .12 & 775 \\
\hline El Manzano & Malleco & 3MAN1A & 37.47 .45 & 72.51 .34 & 560 \\
\hline Nahuelbuta & Malleco & 3NAH7A & 37.47 .20 & 72.59 .59 & 1290 \\
\hline Laguna Icalma & Cautín & 3ICA4A & 38.49 .53 & 71.23 .40 & 1285 \\
\hline Laguna Icalma & Cautín & 3ICA8A & 38.41 .23 & 71.20 .10 & 1155 \\
\hline Captren & Cautín & 3MEL9A & 38.38 .34 & 71.47 .19 & 1210 \\
\hline Lonquimay & Cautín & 3LON6A & 38.21 .02 & 71.16 .44 & 840 \\
\hline Galletué & Cautín & 3GAL2A & 38.35 .33 & 71.26 .12 & 1140 \\
\hline Pino Hachado & Cautín & 3PIN2A & 38.38 .75 & 70.57 .07 & 1620 \\
\hline Puerto Saavedra & Cautín & $3 \mathrm{SAA} 2 \mathrm{~A}$ & 38.46 .48 & 73.16 .13 & NI \\
\hline Nueva Imperial & Cautín & 3NIM1A & 38.45 .81 & 72.54 .99 & 90 \\
\hline Nitrito & Cautín & 3NIT1A & 38.07 .54 & 71.20 .08 & 805 \\
\hline Sierra Nevada & Cautín & 3SIE1A & 38.26 .93 & 71.22 .80 & 1050 \\
\hline Conguillío & Cautín & 3GUI2A & 38.38 .85 & 71.38 .15 & 1120 \\
\hline Llaima & Cautín & 3LLA1A & 38.41 .24 & 71.49 .48 & 1200 \\
\hline Lago Icalma & Cautín & 3ICA7A & 38.43 .22 & 71.12 .53 & 1120 \\
\hline Conguillío & Cautín & 3MEL5A & 38.45 .94 & 71.37 .93 & 750 \\
\hline Lago Budi & Cautín & 3BUD1A & 38.48 .17 & 73.23 .43 & 80 \\
\hline Cunco & Cautín & 3CUN1A & 38.51 .06 & 71.40 .51 & 410 \\
\hline Lago T. Los Santos & Osorno & 2LTS1A & 41.14 & 72.45 & 229 \\
\hline Paso Card. Samoré & Osorno & 3PUY1A & 40.44 .81 & 72.05 .59 & 1060 \\
\hline Petrohué & Osorno & 93PET1A & 41.08 & 72.16 & NI \\
\hline Puyehue & Osorno & 3PUY5A & 40.42 .90 & 71.56 .66 & 1290 \\
\hline Niebla & Valdivia & 3NIE1A & 39.47 .59 & 73.23 .24 & 30 \\
\hline Tres Chiflones & Valdivia & $3 \mathrm{FLO} 2 \mathrm{~A}$ & 40.02 .80 & 73.10 .49 & 360 \\
\hline Lago Maihue & Valdivia & 3MAI1A & 40.12 .44 & 72.08 .29 & NI \\
\hline Chanquín & Valdivia & 3HAN1A & 39.16 .39 & 73.10 .98 & 50 \\
\hline Nigue & Valdivia & 3NIG1A & 39.19 .12 & 73.11 .53 & 50 \\
\hline Corral & Valdivia & 3COR1A & 39.56 .21 & 73.13 .28 & NI \\
\hline Tres Chiflones & Valdivia & 3FLO1A & 40.02 .80 & 73.10 .49 & 360 \\
\hline Futrono & Valdivia & $30 N O 1 \mathrm{~A}$ & 40.09 .59 & 72.35 .16 & NI \\
\hline Riñinahue & Valdivia & 3RIÑ1A & 40.17 .74 & 72.10 .86 & 80 \\
\hline Río El Salto & Chiloé & 2PAL2A & 43.30 & 71.55 & 183 \\
\hline Palena & Chiloé & 2PAL3A & 43.35 & 71.45 & NI \\
\hline Futaleufú & Chiloé & 2FUT4A & 43.08 & 71.40 & NI \\
\hline Río Camahueto & Chiloé & 2CAM1A & 42.50 & 72.53 & 2 \\
\hline Mar Brava & Chiloé & 2BRA1A & 41.50 & 73.55 & 2 \\
\hline Balmaceda & Aisén & 2BAL1B & 45.52 & 71.51 & 530 \\
\hline La Tapera & Aisén & 2TAP3A & 44.40 & 71.45 & NI \\
\hline La Tapera & Aisén & 2TAP4A & 44.38 & 71.39 & NI \\
\hline Puerto M.Balmaceda & Aisén & 2MAR1A & 43.47 & 72.57 & 0 \\
\hline Mallín Grande & Gral. Carrera & 2MAL2A & 46.08 & 72.06 & 351 \\
\hline
\end{tabular}

${ }^{\mathrm{z}} \mathrm{NI}=$ no information

${ }^{y}$ Accesion analyzed by molecular markers. (I DO NOT SEE ANY FOOTNOTE???)

${ }^{\mathrm{x}} \mathrm{MASL}=$ meters above sea level 


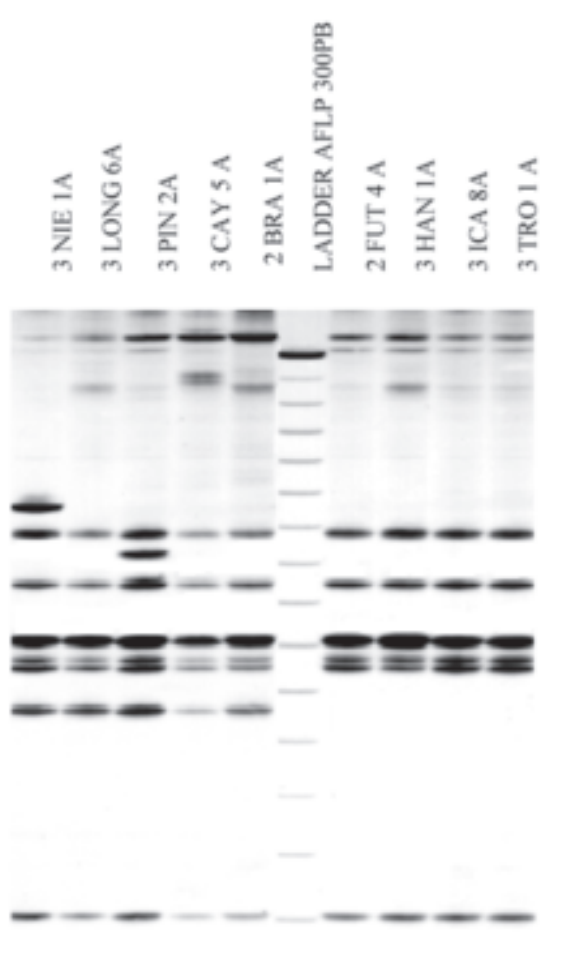

Fig. 1.AFLP banding pattern of Fragaria chiloensis accessions for Eco RI-ACT/ MseI-CAC primer combination.

and $38.5 \mathrm{~cm}$ (Becerra et al., 2001; Maureira et al., 1996).

In the AFLP analysis, 37 polymorphic fragments were generated (Fig. 1), and most of the genotypes clustered around $90 \%$ of similarity, with no apparent regional clusters (Fig. 2). This limited amount of clustering contrasted greatly with the well differentiated phenograms generated using morphological and RAPD data (Becerra et al., 2002; Maureira et al., 1996).

\section{Conclusions}

The wild and cultivated Chilean populations of $F$. chiloensis are highly diverse, based on morphological and molecular markers. Isozymes were not so variable, which agrees with other reports concerning strawberry (Arulsekar et al., 1981). The high level of diversity found in most $F$. chiloensis traits indicates that there is a storehouse of genetic variability available to plant breeders. Because this diversity appears to be widely distributed geographically, the primary considerations in selecting superior parents would appear to be their horticultural characteristics and their environmental adaptations.

\section{Literature Cited}

Arulsekar, S., R. Bringhurst, and V. Voth. 1981. Inheritance of PGI and LAP isozymes in octoploid cultivated strawberries. J. Amer. Soc. Hort. Sci. 106:679-683.

Becerra, V. and P. Gepts. 1994. RFLP diversity of common bean (Phaseolus vulgaris) in its centres of origin. Genome 37:256-263.

Becerra, V., M. Paredes, C. González, and A. Lavin. 2002. Caracterización molecular de 62 posibles progenitores de Fragaria chiloensis (L.) Duch.
Genetic identity (Coefficient of Jaccard)

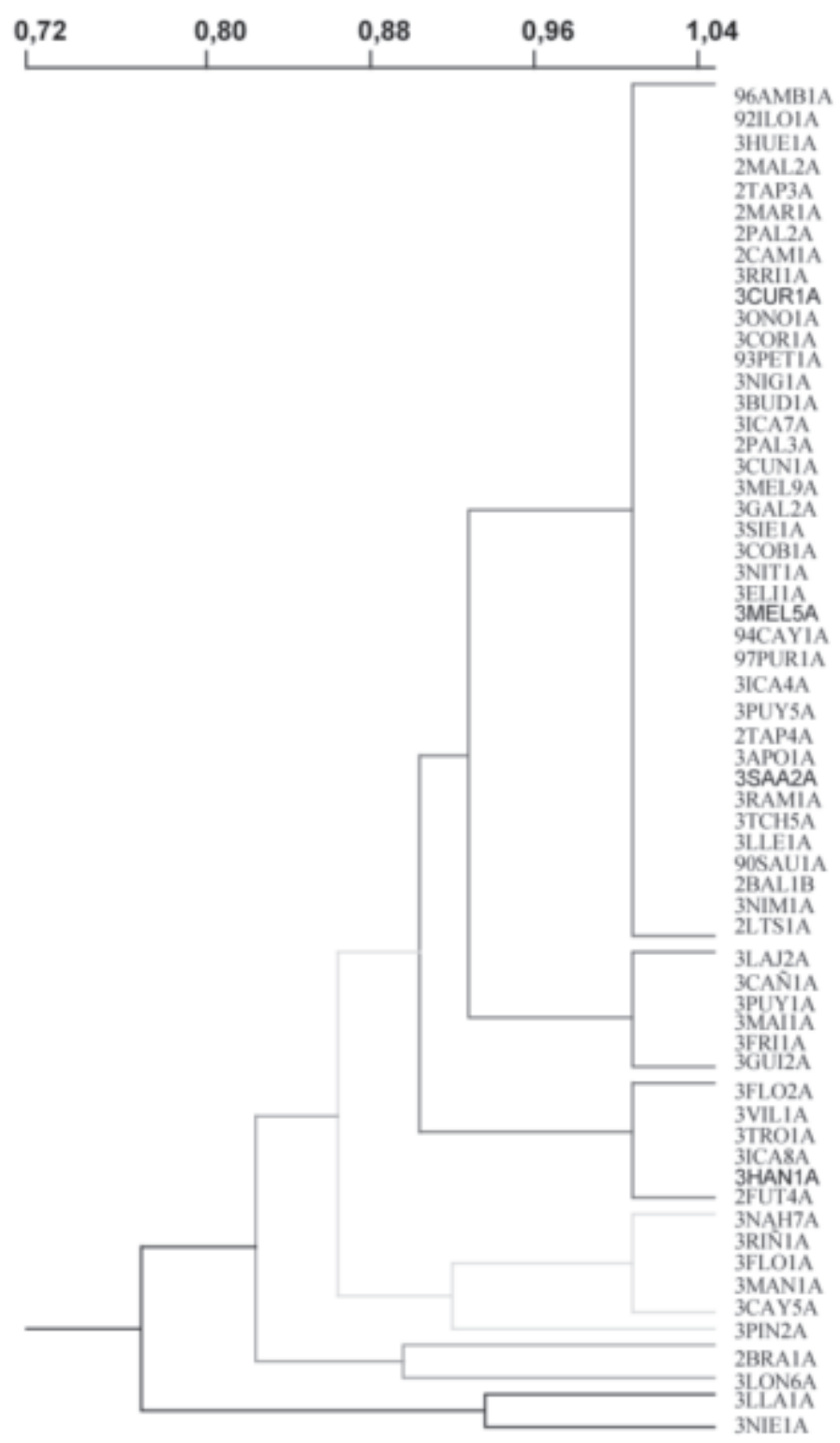

Fig. 2. Genetic relationship among 61 accessions of Fragaria chiloensis, detected by 6 primer combinations of AFLP.

para un programa de mejoramiento.Agro Ciencia 17:145-152.

Becerra, V., M. Paredes, A. Romero, and A. Lavín. 2001. Diversidad bioquímica, molecular y morfológica en frutillas chilenas (Fragaria chiloensis) y sus implicancias en el mejoramiento genético de la especie. Agr. Técnica (Chile) 61:413-428.

Cameron, J., T. Sjulin, J. Ballington, C. Shanks, C. Muñoz, and A. Lavín. 1993. Exploration, collection and evaluation of Chilean Fragaria: Summary of 1990 and 1993 expeditions. Acta Hort. 348:65-74.

Maureira, M., A. Lavín, and A. del Pozo, A. 1996. Caracterización fenotípica y fenológica de siete accesiones chilenas de Fragaria chiloensis (L.) Duch. Agr. Técnica (Chile) 56 (3):201-210.

Vos, P., R. Hogers, M. Bleeker, M. Reijans, T. Van de Lee, M. Hornes, A. Frijters, J. Pot, J. Peleman, M. Kuiper, and M. Zabeau. 1995. AFLP: A new technique for DNA fingerprinting. Nucleic Acids Res. 23(21):4407-4414. 